\title{
Strain-tunable magnetism at ferroelastic domain walls
}

Christensen, D. V.; Frenkel, Y.; Xie, Y. ; Chen, Z.; Hikita, Y. ; Smith, A.; Chen, Y. Z.; Klein, L.; Hwang, H. Y.; Pryds, N.

Total number of authors:

11

Published in:

Nature Physics

Link to article, DOI:

10.1038/s41567-018-0363-x

Publication date:

2019

Document Version

Peer reviewed version

Link back to DTU Orbit

Citation (APA):

Christensen, D. V., Frenkel, Y., Xie, Y., Chen, Z., Hikita, Y., Smith, A., Chen, Y. Z., Klein, L., Hwang, H. Y., Pryds, N., \& Kalisky, B. (2019). Strain-tunable magnetism at ferroelastic domain walls. Nature Physics, 15, 269274. https://doi.org/10.1038/s41567-018-0363-x

\section{General rights}

Copyright and moral rights for the publications made accessible in the public portal are retained by the authors and/or other copyright owners and it is a condition of accessing publications that users recognise and abide by the legal requirements associated with these rights.

- Users may download and print one copy of any publication from the public portal for the purpose of private study or research.

- You may not further distribute the material or use it for any profit-making activity or commercial gain

- You may freely distribute the URL identifying the publication in the public portal 


\title{
Strain tunable magnetism at ferroelastic domain walls
}

\author{
D. V. Christensen ${ }^{1 \dagger}$, Y. Frenkel ${ }^{2 \dagger}, Y . X i e^{3,4,5}$, Z. Chen ${ }^{4}$, Y. Hikita ${ }^{3}$, A. Smith ${ }^{1}$, \\ Y. Z. Chen ${ }^{1}$, L. Klein ${ }^{6}$, H. Y. Hwang ${ }^{3}$, N. Pryds ${ }^{1 *}$, and B. Kalisky ${ }^{2 *}$ \\ ${ }^{1}$ Department of Energy Conversion and Storage, Technical University of Denmark, Ris $\varnothing$ Campus, \\ DK-4000 Roskilde, Denmark. \\ ${ }^{2}$ Department of Physics and Institute of Nanotechnology and Advanced Materials, Bar-Ilan Univer- \\ sity, Ramat-Gan 5290002, Israel. \\ ${ }^{3}$ Stanford Institute for Materials and Energy Sciences, SLAC National Accelerator Laboratory, \\ Menlo Park, California 94025, USA \\ ${ }^{4}$ Department of Applied Physics, Geballe Laboratory for Advanced Materials, Stanford University \\ ${ }^{5}$ Department of Physics, Zhejiang University, Hangzhou, 310027, China \\ ${ }^{6}$ Department of Physics, Nano-magnetism Research Center, Institute of Nanotechnology and Ad- \\ vanced Materials, Bar-Ilan University, Ramat-Gan, 5290002 Israel. \\ 'These authors contributed equally to this work \\ *Email: nipr@dtu.dk and beena@biu.ac.il.
}

\begin{abstract}
:
The ability to write, erase and move domain walls between ferroelastic domains paves the way for making nanoelectronics where the domain wall is the device. Little is, however, known about the magnetic properties of the ferroelastic domain walls. A fascinating model system is $\mathrm{SrTiO}_{3}$ where the ferroelastic domain walls display strain tunable polarity and enhanced conductivity besides being likely to host paired electrons existing both in the superconducting and nonsuperconducting state. Here, we reveal a long-range ferromagnetic order with stripy modulations along the ferroelastic domain walls in $\mathrm{SrTiO}_{3}$ and $\mathrm{SrTiO}_{3}$-based heterointerfaces using a scanning superconducting quantum interference device. The magnetism is coupled to high-mobility itinerant electrons with clear signatures in magnetotransport measurements. Strikingly, the magnetic state is also coupled dynamically to the lattice and can be reversibly tuned by applying local external forces. The study opens up for designing nanoscale devices based on domain walls where strain-tunable ferroelectric, ferroelastic and ferromagnetic orders may coexist.
\end{abstract}


"If $\mathrm{SrTiO}_{3}$ had magnetic properties, a complete study of this material would require a thorough knowledge of all of solid state physics". This statement by M. L. Cohen ${ }^{1}$ succinctly draws attention to the challenges of understanding the physical properties of $\mathrm{SrTiO}_{3}$ (STO). Among its many features are a high electron mobility ${ }^{2}$ and a low carrier density superconducting ground state $^{3,4}$. In the search for properties beyond those found in pristine or doped STO, the coupling between the lattice and electronic degrees of freedom has been utilized successfully: Straining STO has led to a transition into ferroelectricity ${ }^{5}$ and a $300 \%$ increase of the electron mobility ${ }^{6}$. By breaking the lattice symmetry of bulk STO crystals by interfacing STO with e.g. $\mathrm{LaAlO}_{3}$ ( $\mathrm{LAO}$ ), a confined electron gas can be formed, leading to gate-tunability of a wide range of properties ${ }^{7}$. An additional intrinsic lattice symmetry breaking occurs below $\sim 105 \mathrm{~K}$ when the cubic unit cell of STO becomes tetragonal with the long axis along either the [001], [010] or [100] direction ${ }^{8-11}$. In bulk STO, orienting the long axis in each of the three crystallographic directions results in equally stable tetragonal domains, which are separated by domain walls with well-defined orientations and modified electronic properties ${ }^{12,13}$. Thermal cycles of STO above 105K has been observed to result in new configurations of tetragonal domains ${ }^{12,14,15}$. Applied mechanical stresses moves the domain walls by enlarging favorable domains resulting in a hysteretic stress/strain response characteristic for ferroelastic materials. The position of such ferroelastic domain walls can also be conveniently controlled by electric fields, which allows for regulating the electronic properties of LAO/STO at the nanoscale ${ }^{12,13}$.

Surprisingly, magnetic islands were imaged in the LAO/STO heterostructure with LAO thicknesses of at least 3 unit cells ${ }^{16}$. Later, it was shown that the magnitude and orientation of the magnetization on the islands can change in response to local stress ${ }^{17}$. A new dimension was hereby added to the functionalities of STO as spin and its coupling to charge and lattice can be utilized. However, the spin state of the confined electron gas turned out to display some intriguing scientific puzzles that continue to challenge our current understanding of physics on a fundamental level: First, the ferromagnetic order coexists with superconducting order despite ferromagnetism occurring by alignment of spins whereas electrons pair in a singlet state to form the conventional superconducting state $^{18,19}$. Second, the superconductivity can be broken by application of a magnetic field, but the pairing of the electrons can survive in a non-superconducting state ${ }^{20}$. A re- cent experiment suggests that the electron pairing occurs at the ferroelastic domain walls ${ }^{21}$ where they form a nonsuperconducting singlet state ${ }^{20}$ or possibly even a spinpolarized state with more than two paired electrons. The magnetic state and the electron pairing are therefore intimately linked ${ }^{22}$. Despite years of intensive research the magnetic state remains one of the properties of the heterointerface which is poorest understood, hardest to reproduce and most challenging to control.

Several theoretical models have been proposed, including spiral magnetism in a charge-ordered state ${ }^{23}$ and oxygen vacancy induced magnetism ${ }^{24}$. Experimentally, spatially resolved magnetometry measurements have played a key role. A scanning superconducting quantum interference device (SQUID) operating at low temperatures revealed disperse ferromagnetic patches smaller than the $3 \mu \mathrm{m}$ resolution limit ${ }^{16,18}$. Room temperature magnetic force microscopy was also used to probe the magnetic landscape emerging as the electrons at the LAO/STO interface was depleted using a top gate ${ }^{25}$. Additional experimental signatures of magnetism stem from (i) magnetometry measurements without spatial resolution, such as SQUID ${ }^{26}$ \& torque magnetometry ${ }^{19}$, (ii) transport measurements reporting Kondo-like resistance minima ${ }^{27,28}$, anomalous Hall effect ${ }^{29-31}$ \& negative or anisotropic magnetoresistance ${ }^{26,28-30,32,33}$ and (iii) irradiation-based techniques such as $\beta$-decay from nuclear magnetic resonance $^{34} \& X$-ray magnetic circular dichroism ${ }^{35}$. The magnetic state, however, remains poorly understood, and several studies fail to detect the magnetic state using, e.g., scanning SQUID ${ }^{36}$ and neutron reflectometry ${ }^{37}$.

Here, we probe the magnetic state of LAO/STO, $y-\mathrm{Al}_{2} \mathrm{O}_{3}$ (GAO)/STO and bare STO surfaces using scanning SQUID magnetometry. In all cases, we observe a long-range magnetic order with striped modulations oriented parallel to the ferroelastic domain walls of STO. The magnetic state is coupled to the lattice and can be reversibly tuned by applying local external forces. In addition, clear magnetic signatures in the magnetotransport measurements show that the magnetic order is coupled to the itinerant charges. This striking observation opens up for realizing multifunctional nanoelectronics at domain walls with strain-tunable ferromagnetic, ferroelastic and ferroelectric properties.

Imaging striped magnetic order along ferroelastic domain walls: 

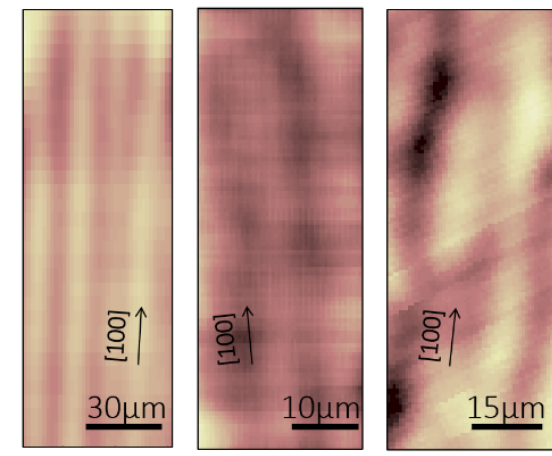

$-1.5$

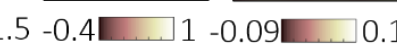

Magnetic flux $\left(m \Phi_{0}\right)$

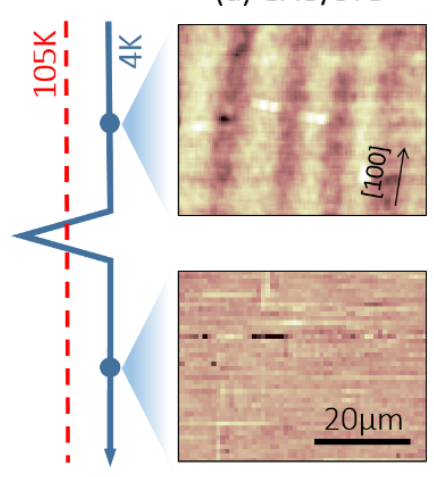

$-1.5 \square-1.5$

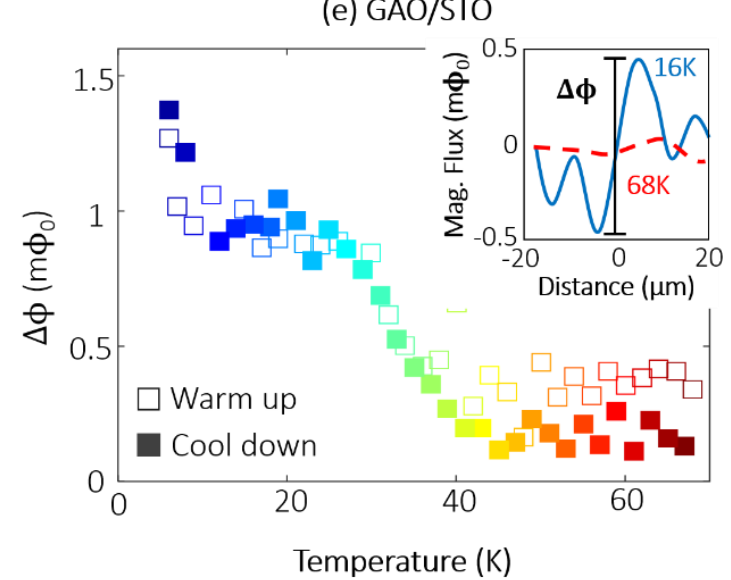

Fig. 1: Scanning SQUID maps of striped magnetic order. (a-c) Large area scans showing magnetic stripes along the [100] crystallographic direction in $\mathrm{\gamma}-\mathrm{Al}_{2} \mathrm{O}_{3}(\mathrm{GAO}) / \mathrm{SrTiO}_{3}(\mathrm{STO}), \mathrm{LaAlO}_{3}(\mathrm{LAO}) / \mathrm{SrTiO}_{3}$ (STO) and vacuum-annealed $\mathrm{SrTiO}_{3}$. (d) The same location on GAO/STO imaged in two subsequent scans before and after thermal cycling the sample above $105 \mathrm{~K}$ (the structural transition temperature of STO). (e) Temperature dependence of the peak-to-peak modulation amplitude $(\Delta \phi)$ defined using line scans as shown in the inset. The modulations disappear as the temperature exceeds $40 \mathrm{~K}$, and reappear without noticeable hysteresis during the subsequent cool down below $40 \mathrm{~K}$. The magnetic flux is offset by the average value of the scan for all figures.

We map the microscopic landscape of the magnetic state in the STO-based systems using scanning SQUID microscopy (see Suppl. Section 1 for materials and methods). The scanning SQUID measures the magnetic flux entering a $1.8 \mu \mathrm{m}$ wide pick-up loop, and produces a twodimensional map of the flux when the pick-up loop scans across the sample surface. The measurements are performed in the absence of an externally applied magnetic field (background field $<0.1 \mu \mathrm{T}$ ) and therefore probe the magnetic field originating from a spontaneous magnetic order in the sample. In the case of LAO/STO, such measurements originally showed the presence of ferromagnetic patches ${ }^{16}$ and their coexistence with superconductivity ${ }^{18}$. Looking into regions without ferromagnetic patches and enhancing the signal-to-noise ratio, our magnetic flux maps reveal wide, striped modulations in both LAO/STO, GAO/STO and vacuum-annealed STO (see Figure 1a-c). The striped modulations extend over hundred micrometers showing a long-range magnetic order. The striped modulations in the magnetic flux have been observed in five GAO/STO heterostructures with a typical magnitude on the order of $1 \mathrm{~m} \phi_{0}$ at $5 \mathrm{~K}$. The modulations are generally stronger in GAO/STO compared to LAO/STO and STO. As the magnetic flux escaping a homogeneously magnetized sample will show a homogeneous flux map in regions far from the sample edges, the flux modulations we observe point to a magnetization with spatial inhomogeneities. We estimate that the typical modulation magnitude of $\sim 1 \mathrm{~m} \phi_{0}$ corresponds to changes in the magnetization on the order of $0.05 \mu_{\mathrm{B}}$ pr. surface unit cell (see Suppl. Section 2). The inhomogeneities are observed along the [100], [010], [110] and [110] crystallographic directions of STO in all three material systems. Remarkably, these orientations coincide with the orientation of ferroelastic domain walls on the (001) surface of STO ${ }^{9,13}$. When we cycle the temperature above $105 \mathrm{~K}$ in the case of
GAO/STO we observe that a set of [100]-oriented striped magnetic modulations with an amplitude of $0.5 \mathrm{~m} \phi_{0}$ changes to barely detectable [110]-oriented modulations with an amplitude of less than $0.05 \mathrm{~m} \phi_{0}$ (see Figure 1d). Changes in the striped magnetic landscape after thermal cycling also occur for bare STO without the GAO layer (see Suppl. Section 3). The results of the thermal cycling eliminate that the magnetic stripes are caused by a trivial magnetic contamination.

Scanning SQUID has also been used to map the spatial distribution of current flow in $\mathrm{LAO} / \mathrm{STO}^{12}$ and $\mathrm{GAO} / \mathrm{STO}^{38}$, which revealed striped current modulations with the same crystallographic orientations as the magnetic stripes. Comparing the current flow with optical imaging of the domain structure furthermore showed that their orientations coincide ${ }^{14}$. Here, we perform magnetometry and current mapping on the same area, and observe a clear relation between the magnetic and current modulations (see Suppl. Section 4), implying that both the magnetic and current modulations are related to the domain structure.

When the temperature is raised gradually from $5 \mathrm{~K}$ to 70 $\mathrm{K}$, the magnetic modulations monotonically decrease in size until their disappearance at around $40 \mathrm{~K}$ (see Figure 1e). This characteristic temperature also roughly marks another anomaly of the STO substrate where its quantum paraelectric transition causes the dielectric constant to diverge ${ }^{39,40}$. Other interesting observations below this temperature are polarity at the STO domain walls ${ }^{41,42}$ and striped modulations in the current flow. Cooling the GAO/STO heterostructure to $5 \mathrm{~K}$ again reintroduces the magnetic modulations without noticeable changes in size or location as long as the $105 \mathrm{~K}$ transition temperature is not crossed.

\section{Coupling between the magnetic state and current flow:}


The disappearance of the magnetic modulations above $40 \mathrm{~K}$ is reflected in the Hall and magnetoresistance measurements as observed particularly clearly in the case of GAO/STO. A Hall effect which is linear as a function of the magnetic field serves as a valuable tool for extracting the carrier density in a single-band conductor, while deviations from linearity can be used to deduce the band structure or magnetism. In the case of $\mathrm{LAO} / \mathrm{STO}^{43}$, the Hall resistance is S-shaped at low temperatures for carrier densities exceeding $\sim 1.7 \cdot 10^{13} \mathrm{~cm}^{-2}$. The S-shape originates from transport occurring in two $n$-type bands with $d_{x y}$ and $d_{x z} / d_{y z}$ character $^{43}$. The presence of magnetism can lead to the anomalous Hall effect, which has been observed as small perturbations to the S-shape ${ }^{29-31}$. In the case of GAO/STO, the Hall resistance $\left(R_{x y}\right)$ is linear for $T>40 \mathrm{~K}$ revealing, for the sample presented in Figure $2 \mathrm{a}$, a high carrier density of $n_{s} \sim 6 \cdot 10^{14} \mathrm{~cm}^{-2}$. For $T<40 \mathrm{~K}$, pronounced nonlinearities arise without hysteresis. At these low temperatures, $\left|d R_{x y} / d B\right|$ increases at high magnetic fields, opposite to the $\mathrm{S}$-shaped non-linearities observed in LAO/STO. If the nonlinearity of the Hall resistance in GAO/STO were due to two-band conductivity, it would entail a coexistence of a large number of electrons and holes, which we reject as detailed in Suppl. Section 5 . Instead, we attribute the non-linearity to the anomalous Hall effect resistance $\left(R_{x y}^{A H E}\right)$ arising from the interaction between the itinerant electrons and the component of the magnetization normal to the interface ${ }^{44}, R_{x y}^{A H E}=$ $R_{S}^{A H E} M_{z}$. We find that the anomalous Hall prefactor $\left(R_{S}^{A H E}\right)$ scales linearly with the sheet resistance $\left(R_{S}\right)$ as expected when skew scattering mechanism dominates ${ }^{44}$ (see Suppl. Section 6). This leads to the following expression

$$
R_{x y}=R_{x y}^{O H E}+R_{x y}^{A H E}=-\frac{1}{e n_{s}} B+k R_{S}(B, T) M_{z}(B, T)
$$

where $R_{S}^{O H E}$ is the ordinary Hall effect and $k$ is a field and temperature independent constant. For GAO/STO the $d_{x z} / d_{y z}$ bands have been reported ${ }^{45}$ to be comparable or even lower in energy than $d_{x y}$. This, together with the high density of states of the $d_{x z} / d_{y z}$ bands, means that the majority of the electrons are expected to populate the $d_{x z} / d_{y z}$ bands. For simplicity we describe the ordinary Hall effect through the sheet carrier density $\left(n_{s}\right)$ with a one band model. Our data cannot exclude the presence of a second $n$-type band, but as outlined in Suppl. Section 6 this will not change the conclusions drawn here. At high magnetic fields, the sheet resistance increases linearly with the magnetic field, which, remarkably, results in a non-saturating anomalous Hall effect up to at least $15 \mathrm{~T}$ (see Figure $2 \mathrm{~b}$ ). The usual extraction of the carrier density from the slope of the Hall resistance at high magnetic fields is therefore invalid as this region includes contributions from both the ordinary and anomalous Hall effect. The carrier densities can be most accurately determined

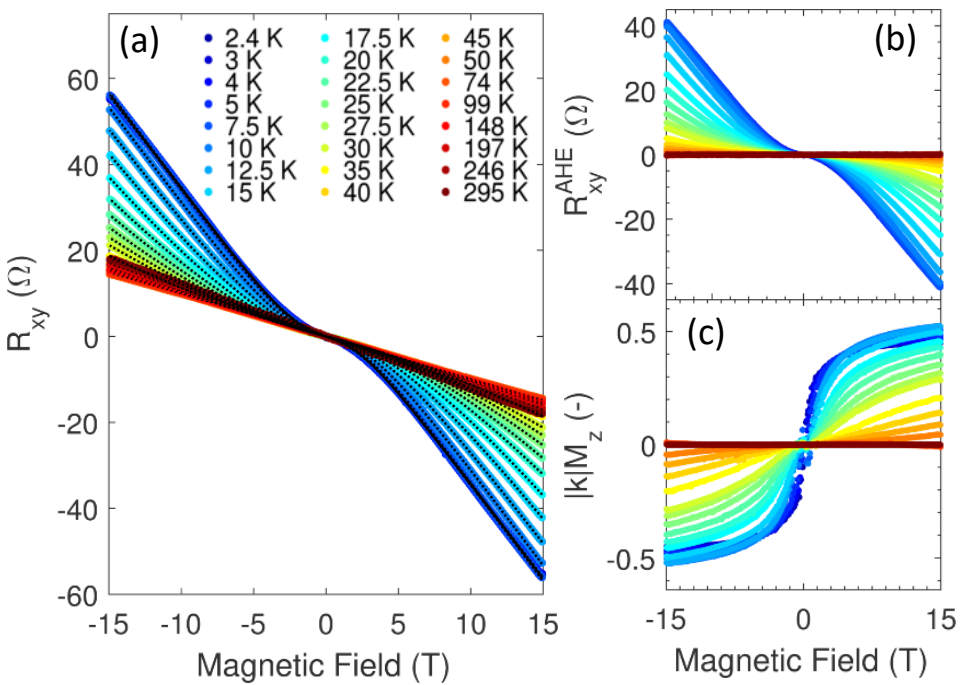

Fig. 2: Anomalous Hall effect. (a) Hall resistance $\left(R_{x y}\right)$ as a function of the magnetic field applied perpendicular to the interface of GAO/STO. Non-linearities are observed below $40 \mathrm{~K}$. (b) Anomalous Hall resistance $\left(R_{x y}^{A H E}\right)$ obtained from $R_{x y}$ by subtracting the ordinary Hall effect determined from the low-field slope of $R_{x y}$. (c) The component of the magnetization perpendicular to the interface $\left(M_{z}\right)$ multiplied by a proportionality constant $k$ as described in the main text.

at low magnetic fields where the contribution from the anomalous Hall effect is small (see Suppl. Section 6). The low-field slope of the Hall resistance is constant at all temperatures (see Figure 2a) and we can therefore extract a temperature-independent carrier density of $\sim 6 \cdot 10^{14} \mathrm{~cm}^{-2}$. This is consistent with the temperature independent carrier density deduced optically from the infrared Berreman mode similar to Ref. 46.

By extracting $k M_{z}(B, T)=\left(R_{x y}-R_{x y}^{O H E}\right) / R_{S}$ we infer that the component of the magnetization parallel to the perpendicular magnetic field starts to saturate at $\sim 2 \mathrm{~T}$ for temperatures below $\sim 8 \mathrm{~K}$ (see Figure $2 \mathrm{c}$ ). Increasing the temperature opposes the alignment of magnetic moments up to $T \sim 40 \mathrm{~K}$ where the magnetization is no longer detectable. This agrees well with the disappearance of the magnetic modulations observed with scanning SQUID. Together with the appearance of magnetic modulations and non-linear Hall resistances for $T<40 \mathrm{~K}$, we also observe that the magnetoresistance deviates from Kohler's rule $^{47}$ at these temperatures. This is consistent with the fact that Kohler's rule does not consider the reduction of charge carrier scattering due to field-induced suppression of spin fluctuations (see Suppl. Section 6). A similar behavior of the Hall resistance and magnetoresistance is observed in four samples (see Suppl. Section 6-7).

\section{Mechanical tuning of the magnetic state:}

A particularly intriguing feature of the magnetic stripes observed is their tunability under small stresses. When applying a constant force to GAO/STO using the scanning SQUID probe, the temperature dependence of the magnetic modulations is markedly different (see Figure 3a). 
(a) Temperature dep. at $56 \mathrm{nN}$

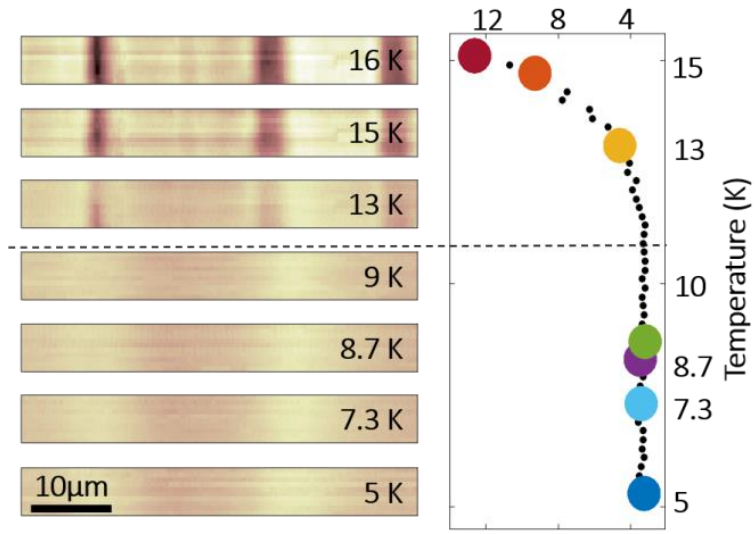

Magnetic flux $\left(m \Phi_{0}\right)-6.8 \square 6.8$ (b) Pressure dep. at $15 \mathrm{~K}$
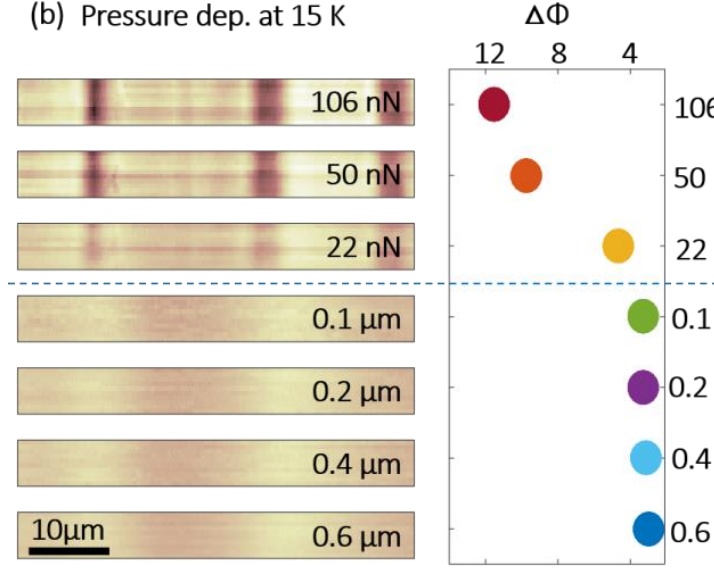

Magnetic flux $\left(m \Phi_{0}\right)-6.8$

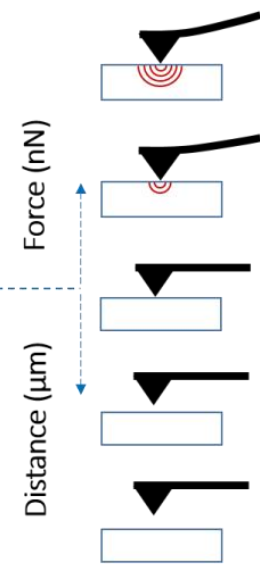

Fig. 3: Pressure dependent magnetic signal. (a) Spatial modulation in the magnetic flux as a function of temperature while pushing the tip of the SQUID into the GAO/STO sample with a force of $56 \mathrm{nN}$. Above $\sim 10 \mathrm{~K}$ the magnetic modulations become very sharp and the peak-to-peak modulation amplitude $(\Delta \phi)$ increases monotonically with temperature. (b) Magnetic modulations at a fixed temperature of $15 \mathrm{~K}$ as the SQUID tip approaches the GAO/STO sample. When the SQUID reaches the sample, sharp magnetic modulations emerge. Further lowering the SQUID results in applying a stronger external force to the sample, which causes an increase in the peak-to-peak modulation amplitude. The force is measured capacitatively via the deflection of the SQUID cantilever. In all cases, the magnetic flux is offset by the average value of the scan.

Instead of the gradual decrease of the modulation strength observed up to $40 \mathrm{~K}$, sharp, stripy features emerge when a force of $56 \mathrm{nN}$ is applied. This applied force corresponds to a pressure on the order of $10^{7} \mathrm{~Pa}$ (see Methods). The features start to emerge around $10 \mathrm{~K}$ and the flux modulations are increased by a factor of four when reaching $16 \mathrm{~K}$. The sharp features are only obtained after applying a local force to the sample surface with the probe, and the features become monotonically stronger when increasing the applied force (see Figure $3 \mathrm{~b}$ ). Interestingly, in all cases, the original magnetic landscape without the sharp magnetic modulations can be restored without noticeable hysteresis by lowering the temperature or removing the force. A similar behavior is also observed in LAO/STO (see Suppl. Section 8). We emphasize that this observation is different from the report of Kalisky et al. ${ }^{17}$, where they imaged and reoriented the magnetization of magnetic islands in LAO/STO by applying local stress with a scanning probe. The signal of the magnetic islands was almost two orders of magnitude stronger, they appeared in isolated and dispersed locations, and did not change with temperature ${ }^{16}$. The magnetic stripes we observe here are weaker, long range in nature, become stronger with temperature under the application of stress, and appear at different locations after temperature cycles. The relation between stress and magnetism can also be examined by investigating the naturally occurring stresses in the samples by mapping large areas. We find that areas with complex domain structures, formed to relieve local stress in the sample, are more likely to exhibit magnetic stripes (such as Figure 1a-c). In the samples measured in this study, there were large areas with an extent of hundreds of microns, where no magnetic stripes were observed within our detection limit of 0.05 $m \phi_{0}$ (d.c.). After temperature cycles above $105 \mathrm{~K}$, magnetic stripes can be found within these areas as in Figure $2 b$. Modulations are observed more strongly in the vicinity of areas expected to exhibit stress such as close to sample borders or around a scratch introduced intentionally on the surface of one sample. Concurrent with higher stresses, these areas may also attact the largest amount of defects such as oxygen vacancies.

We further measure the magnetic response (susceptibility) by applying a small oscillating magnetic field of \pm 0.3 $\mathrm{mT}$ locally to the GAO/STO heterostructure while simultaneously applying a local force of $56 \mathrm{nN}$ with the scanning probe. We find that the weak magnetic susceptibility observed below $5 \mathrm{~K}$ increases markedly above $\sim 8 \mathrm{~K}$ (see Figure $4 a$ ) and reaches a strong paramagnetic response up to $8 \Phi_{0} / \mathrm{A}$ at $16 \mathrm{~K}$. The spatially resolved susceptibility data (Suppl. Section 9) reveals that the areas in Figure 3 with strong magnetic signals also show a slower increase in the paramagnetic susceptibility above $\sim 8 \mathrm{~K}$. Interestingly, this characteristic temperature also appears in the anomalous Hall effect analysis. The characteristic magnetic field $\left(B_{c}\right)$ for the emergence of the anomalous Hall resistance can be described by the half width at half maximum of the bell-shaped $\mathrm{d} R_{x y} / \mathrm{d} B$. Below $\sim 8 \mathrm{~K}$, the characteristic field is temperature independent, consistent with reports on $\mathrm{NGO} / \mathrm{STO}^{31}$, as expected if an exchange coupling in a magnetically ordered state prevents the magnetization to be induced or aligned along the field (see Figure 4a). At higher temperatures, $B_{c}$ is linearly increasing with temperature pointing towards a behavior similar to paramagnetism where the alignment of magnetic moments is countered by thermal fluctuations.

\section{Discussion:}



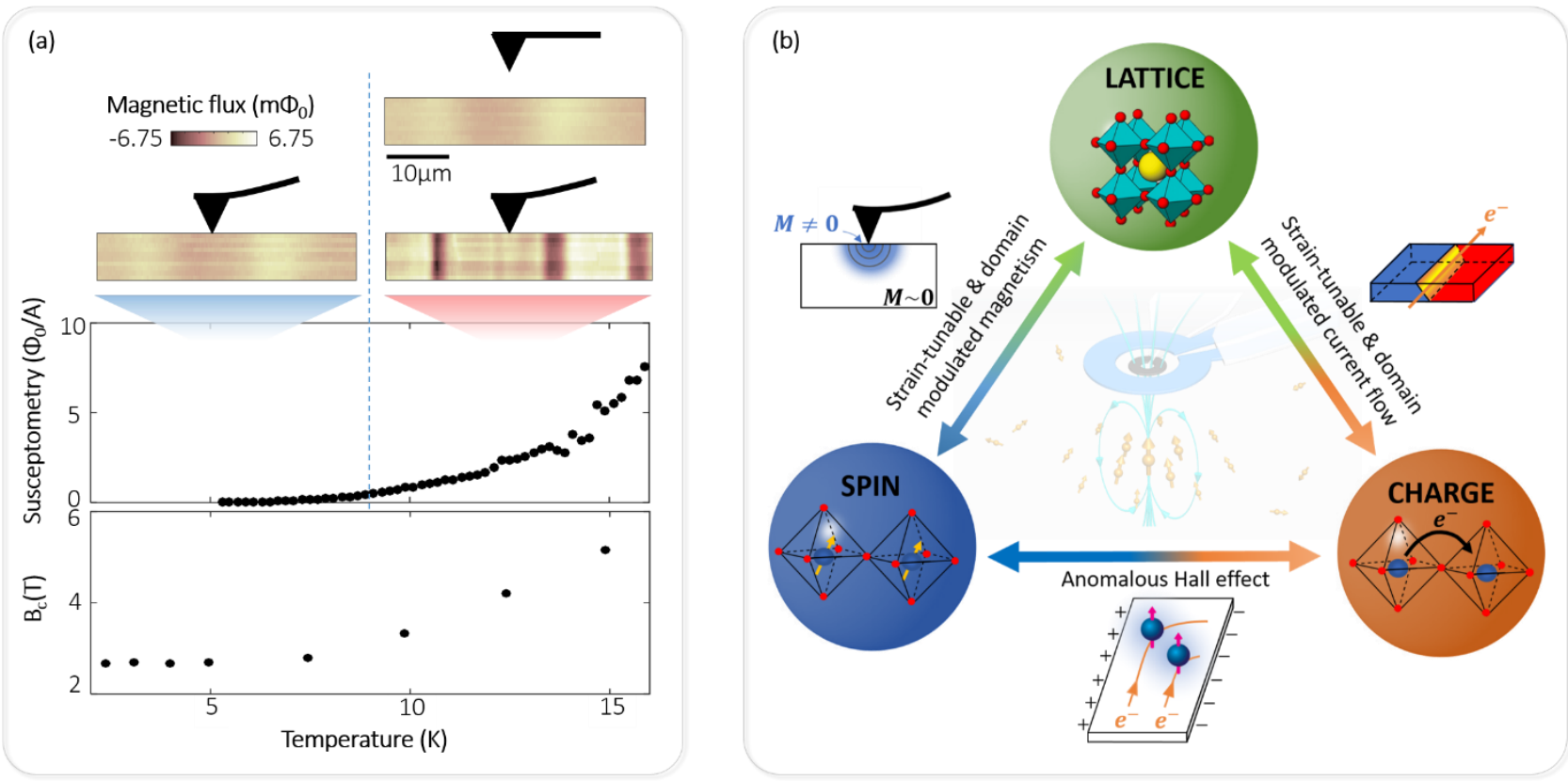

Fig. 4: Coupling between lattice, spin and charge. (a) Temperature dependence of the magnetic properties in GAO/STO showing (i) emergence of sharp magnetic features above $\sim 10 \mathrm{~K}$ upon application of an external force, (ii) an increase in the magnetic susceptibility with an onset of $\sim 8 \mathrm{~K}$ and (iii) the characteristic field $B_{c}$, defined as the half width at half maximum of $\mathrm{d} R_{x y} / \mathrm{d} B$ from Figure $2 \mathrm{a}$, undergoing a change from temperature-independent to linearly increasing with temperature at $\sim 8 \mathrm{~K}$. (b) $\mathrm{A}$ close coupling between the lattice, spin and charge degrees of freedom. The magnetic state is closely coupled to the lattice as revealed by applying external force locally to the sample with the scanning probe. The tetragonal domain formation also causes local lattice distortions, which both favors ferromagnetic order and modulates the charge current. The charge current is, in turn, scattered asymmetrically by the magnetic moments, giving rise to the anomalous Hall resistance.

Evidence of ferromagnetism in STO-based heterostructures has been reported by several independent experimental techniques ${ }^{22}$. Despite theoretical and experimental efforts, the origin of the unexpected ferromagnetism in these heterostructures is still not clear. Here, we observe a long-range magnetic state that is modulated by the crystallographic domains in STO and is highly tunable by local application of external forces. The striped magnetic modulations originate from the tetragonal domain structure of STO and are thus displayed in both STO and the STO-based heterostructures. The undoped STO studied here is, however, electrically insulating, which hinders spintronic applications and investigation of the magnetic state through the anomalous Hall effect and magnetoresistance. Interfacing STO with GAO or LAO on the other hand provides itinerant carriers to the surface of STO, which couple closely to the magnetic moments. Our observations uncover characteristic temperatures of $\sim 8 \mathrm{~K}$ and $\sim 40 \mathrm{~K}$ occurring in both transport and SQUID measurements. We propose that these characteristic temperature regimes originate from the following reasons:

(1) Below $8 \mathrm{~K}$, the STO-based heterostructures are in a magnetically ordered state, which manifests itself in small or no modulations in magnetization in the scanning SQUID maps (Figure 3a-b). This state is characterized by a very weak (almost zero) susceptibility in response to small magnetic fields of $\pm 0.3 \mathrm{mT}$. Applying a temperature- independent magnetic field of $B_{c} \sim 2 \mathrm{~T}$ aligns the magnetic moments perpendicular to the interface, which causes the anomalous Hall effect (Figure 1a and 4a). At these temperatures, a local application of an external force of up to $106 \mathrm{nN}$ has no observable effect on the ordered state.

(2) Above $\sim 8 \mathrm{~K}$, the magnetic order is less robust. This is supported by the increase in the paramagnetic susceptibility under a small applied force of $56 \mathrm{nN}$ (Figure 4a) suggesting a gradual change where ordered magnetic moments convert into paramagnetic moments. The conversion is slower at the domain walls where the magnetic moments appear to be ordered more strongly, leading to areas with a slower growth in the magnetic susceptibility and large modulations of the magnetic signal (Suppl. Section 9). The alignment of the paramagnetic moments with the magnetic field is countered by thermal fluctuations resulting in a linear increase of $B_{c}$ with temperature (see Figure 4a). The fragile magnetic order above $8 \mathrm{~K}$ is highly sensitive to externally applied forces, and stronger magnetic modulations can be induced here (Figure 4a). This points towards a delicate balance between the unperturbed magnetic order existing in the absence of stress and a ferromagnetic order induced by the stress. Applying force causes a shift towards the ferromagnetic order in this temperature range. One of the possible mechanisms for the appearance of ferromagnetism is that the magnetic moments are produced by oxygen vacancies ${ }^{24,48}$. This is 
supported by the vast number of oxygen vacancies present particularly in GAO/STO ${ }^{49,50}$ as well as the localized in-gap states formed by the oxygen vacancies ${ }^{51}$.

The unperturbed magnetic order may be due to e.g. spiral magnetic ordering ${ }^{23}$ or electron pairing ${ }^{20,22}$ where it is expected that the state is non-hysteretic and result in a small magnetic susceptibility when applying small magnetic fields. Strikingly, it has been predicted that a pressure-sensitive transition from a spiral order to a ferromagnetic order occurs due to a polar distortion in STO where titanium ions are displaced relative to the oxygen ions $^{23}$. This agrees well with recent experiments in LAO/STO showing that applying a local external force changes the polar state at the ferroelastic domain walls of $\mathrm{STO}^{42}$. Such a polarity-induced ferromagnetic order may also explain why the magnetic stripes (even in absence of an external force) are parallel to the domain walls in STO, as the domain walls have been observed to be polar ${ }^{41,42}$.

(3) Above $40 \mathrm{~K}$, all signatures of magnetism from anomalous Hall effect, magnetoresistance and scanning SQUID vanish. This characteristic temperature also marks the disappearance of the striped current modulations in $\mathrm{LAO} / \mathrm{STO}^{12}$ as well as the disappearance of magnetism in LAO/STO according to some studies ${ }^{32,33}$. It also agrees reasonably well with the emergence of polar domain walls ${ }^{41,42}$ only below approximately $50 \mathrm{~K}$, again hinting towards the influence of STO polarity.

The advances made here demonstrate that the STO-based material systems show an intriguing link between the charge, spin and lattice degrees of freedom in STO-based heterostructures as illustrated in Figure $4 \mathrm{~b}$. The magnetic state couples to the current flow causing an asymmetric scattering of the charge carriers (Figure 2). The lattice and its domains also highly influence both the magnetic state (Figure 1) and current flow $12,38,42$. In particular, applying an external pressure in vicinity of the ferroelastic domain walls dramatically alters the magnetic state (Figure 3 and Suppl. Section 9), the current flow ${ }^{42}$ and the polarity of the ferroelastic domain walls ${ }^{42}$. We therefore propose that an exciting multiferroic state awaits to be discovered where a strain-tunable ferroelectric, ferroelastic and ferromagnetic order coexists. The key role played by the ferroelastic domain walls of STO further demonstrates the prospects of using domain walls for functional nanoelectronics with tunable magnetic properties. STO proves, once again, to be an exciting multifunctional material that truly requires 'a thorough knowledge of all of solid state physics'.

\section{Methods:}

See Suppl. Section 1.

\section{Data availability:}

The data that support the plots within this paper and other findings of this study are available from the corresponding authors upon reasonable request.

\section{References:}

1. Cohen, M. L. Superconductivity in Low-Carrier-Density Systems: Degenerate Semiconductors. Supercond. Part I 615 (1969).

2. Frederikse, H. P. R. \& Hosler, W. R. Hall Mobility in $\mathrm{SrTiO}_{3}$. Phys. Rev. 161, 822 (1967).

3. Schooley, J. F., Hosler, W. R. \& Cohen, M. L. Superconductivity in Semiconducting $\mathrm{SrTiO}_{3}$. Phys Rev Lett 12, 474-475 (1964).

4. Schooley, J. F. et al. Dependence of the Superconducting Transition Temperature on Carrier Concentration in Semiconducting $\mathrm{SrTiO}_{3}$. Phys Rev Lett 14, 305-307 (1965).

5. Haeni, J. H. et al. Room-temperature ferroelectricity in strained $\mathrm{SrTiO}_{3}$. Nature 430, 758 (2004).

6. Jalan, B., Allen, S. J., Beltz, G. E., Moetakef, P. \& Stemmer, S. Enhancing the electron mobility of $\mathrm{SrTiO}_{3}$ with strain. Appl. Phys. Lett. 98, 132102 (2011).

7. Pai, Y.-Y., Tylan-Tyler, A., Irvin, P. \& Levy, J. Physics of $\mathrm{SrTiO}_{3}$ based heterostructures and nanostructures: a review. Rep. Prog. Phys. 81, 036503 (2018).

8. Rimai, L. Electron paramagnetic resonance of trivalent gadolinium ions in strontium and barium titanates. Phys. Rev. 127, 702 (1962).

9. Cowley, R. A. Lattice Dynamics and Phase Transitions of Strontium Titanate. Phys. Rev. 134, A981-A997 (1964).

10. Heidemann, A. \& Wettengel, H. Die Messung der Gitterparameteränderung von $\mathrm{SrTiO}_{3}$. Z. Für Phys. Hadrons Nucl. 258, 429-438 (1973).

11. Lytle, F. W. X-Ray Diffractometry of Low-Temperature Phase Transformations in Strontium Titanate. J. Appl. Phys. 35, 2212 (1964).

12. Kalisky, B. et al. Locally enhanced conductivity due to the tetragonal domain structure in $\mathrm{LaAlO}_{3} / \mathrm{SrTiO}_{3}$ heterointerfaces. Nat. Mater. 1-5 (2013). doi:10.1038/nmat3753

13. Honig, M. et al. Local electrostatic imaging of striped domain order in $\mathrm{LaAlO}_{3} / \mathrm{SrTiO}_{3}$. Nat. Mater. 12, 1112-1118 (2013).

14. Erlich, Z. et al. Optical Study of Tetragonal Domains in LaA$\mathrm{IO}_{3} / \mathrm{SrTiO}_{3}$. J. Supercond. Nov. Magn. 28, 1017-1020 (2015).

15. Frenkel, Y. et al. Anisotropic Transport at the $\mathrm{LaAlO}_{3} / \mathrm{SrTiO}_{3}$ Interface Explained by Microscopic Imaging of Channel-Flow over $\mathrm{SrTiO}_{3}$ Domains. ACS Appl. Mater. Interfaces 8, 12514-12519 (2016).

16. Kalisky, B. et al. Critical thickness for ferromagnetism in LaA$\mathrm{IO}_{3} / \mathrm{SrTiO}_{3}$ heterostructures. Nat. Commun. 3, 922 (2012).

17. Kalisky, B. et al. Scanning Probe Manipulation of Magnetism at the $\mathrm{LaAlO}_{3} / \mathrm{SrTiO}_{3}$ Heterointerface. Nano Lett. 12, 4055-4059 (2012).

18. Bert, J. A. et al. Direct imaging of the coexistence of ferromagnetism and superconductivity at the $\mathrm{LaAlO}_{3} / \mathrm{SrTiO}_{3}$ interface. Nat. Phys. 7, 767-771 (2011).

19. Li, L., Richter, C., Mannhart, J. \& Ashoori, R. C. Coexistence of magnetic order and two-dimensional superconductivity at LaA$\mathrm{IO}_{3} / \mathrm{SrTiO}_{3}$ interfaces. Nat. Phys. 7, 762-766 (2011).

20. Cheng, G. et al. Electron pairing without superconductivity. Nature 521, 196-199 (2015).

21. Pai, Y.-Y. et al. One-Dimensional Nature of Pairing and Superconductivity at the $\mathrm{SrTiO}_{3} / \mathrm{LaAlO}<\mathrm{sub}>3$ Interface. Phys. Rev. Lett. 120, 147001 (2018).

22. Pai, Y.-Y., Tylan-Tyler, A., Irvin, P. \& Levy, J. $\mathrm{LaAlO}_{3} / \mathrm{SrTiO}_{3}$ : a tale of two magnetisms. ArXiv Prepr. ArXiv161000789 (2016).

23. Banerjee, S., Erten, O. \& Randeria, M. Ferromagnetic exchange, spin-orbit coupling and spiral magnetism at the $\mathrm{LaAlO}_{3} / \mathrm{SrTiO}_{3}$ interface. Nat. Phys. 9, 626-630 (2013).

24. Pavlenko, N., Kopp, T., Tsymbal, E. Y., Sawatzky, G. A. \& Mannhart, J. Magnetic and superconducting phases at the LaA- 
$\mathrm{IO}_{3} / \mathrm{SrTiO}_{3}$ interface: The role of interfacial Ti 3d electrons. Phys. Rev. B 85, 020407 (2012).

25. Bi, F. et al. Room-temperature electronically-controlled ferromagnetism at the $\mathrm{LaAlO}_{3} / \mathrm{SrTiO}_{3}$ interface. Nat. Commun. 5, 5019 (2014).

26. Ariando et al. Electronic phase separation at the $\mathrm{LaAlO}_{3} / \mathrm{SrTiO}_{3}$ interface. Nat. Commun. 2, 188 (2011).

27. Brinkman, A. et al. Magnetic effects at the interface between non-magnetic oxides. Nat. Mater. 6, 493-496 (2007).

28. Hu, H.-L. et al. Subtle Interplay between Localized Magnetic Moments and Itinerant Electrons in $\mathrm{LaAlO}_{3} / \mathrm{SrTiO}_{3}$ Heterostructures. ACS Appl. Mater. Interfaces 8, 13630-13636 (2016).

29. Joshua, A., Ruhman, J., Pecker, S., Altman, E. \& Ilani, S. Gatetunable polarized phase of two-dimensional electrons at the LaA$\mathrm{IO}_{3} / \mathrm{SrTiO}_{3}$ interface. Proc. Natl. Acad. Sci. 110, 9633-9638 (2013).

30. Seri, S., Schultz, M. \& Klein, L. Interplay between sheet resistance increase and magnetotransport properties in $\mathrm{LaAlO}_{3} / \mathrm{SrTiO}_{3}$. Phys. Rev. B 86, 085118 (2012).

31. Gunkel, F. et al. Defect Control of Conventional and Anomalous Electron Transport at Complex Oxide Interfaces. Phys. Rev. X 6, 031035 (2016).

32. Ben Shalom, M. et al. Anisotropic magnetotransport at the $\mathrm{SrTiO}_{3} / \mathrm{LaAlO}_{3}$ interface. Phys. Rev. B 80, 140403 (2009).

33. Wang, X. et al. Magnetoresistance of two-dimensional and threedimensional electron gas in $\mathrm{LaAlO}_{3} / \mathrm{SrTiO}_{3}$ heterostructures: Influence of magnetic ordering, interface scattering, and dimensionality. Phys. Rev. B 84, 075312 (2011).

34. Salman, Z. et al. Nature of Weak Magnetism in $\mathrm{SrTiO}_{3} / \mathrm{LaAlO}_{3}$ Multilayers. Phys. Rev. Lett. 109, 257207 (2012).

35. Lee, J.-S. et al. Titanium $d_{x y}$ ferromagnetism at the $\mathrm{LaAlO}_{3} / \mathrm{SrTiO}_{3}$ interface. Nat. Mater. 1-4 (2013). doi:10.1038/nmat3674

36. Wijnands, T. Scanning superconducting quantum interference device microscopy: sensitive mapping of magnetic flux on thin films. (Scanning superconducting quantum interference device microscopy, University of Twente, 2013).

37. Fitzsimmons, M. R. et al. Upper Limit to Magnetism in LaA$\mathrm{IO}_{3} / \mathrm{SrTiO}_{3}$ Heterostructures. Phys. Rev. Lett. 107, 217201 (2011).

38. Christensen, D. V. et al. Electron Mobility in $\mathrm{\gamma}-\mathrm{Al}_{2} \mathrm{O}_{3} / \mathrm{SrTiO}_{3}$. Phys. Rev. Appl. 9, (2018).

39. Neville, R. C., Hoeneisen, B. \& Mead, C. A. Permittivity of Strontium Titanate. J. Appl. Phys. 43, 2124-2131 (1972).

40. Rowley, S. E. et al. Ferroelectric quantum criticality. Nat. Phys. 10, 367-372 (2014).

41. Scott, J. F., Salje, E. K. H. \& Carpenter, M. A. Domain Wall Damping and Elastic Softening in $\mathrm{SrTiO}_{3}$ : Evidence for Polar Twin Walls. Phys. Rev. Lett. 109, (2012).

42. Frenkel, Y. et al. Imaging and tuning polarity at $\mathrm{SrTiO}_{3}$ domain walls. Nat. Mater. (2017). doi:doi:10.1038/nmat4966

43. Joshua, A., Pecker, S., Ruhman, J., Altman, E. \& Ilani, S. A universal critical density underlying the physics of electrons at the LaA$\mathrm{IO}_{3} / \mathrm{SrTiO}_{3}$ interface. Nat. Commun. 3, 1129 (2012).

44. Nagaosa, N., Sinova, J., Onoda, S., MacDonald, A. H. \& Ong, N. P. Anomalous Hall effect. Rev. Mod. Phys. 82, 1539-1592 (2010).

45. Cao, Y. et al. Anomalous orbital structure in a spinel-perovskite interface. Npj Quantum Mater. 1, 16009 (2016).

46. Yazdi-Rizi, M. et al. Infrared ellipsometry study of the confined electrons in a high-mobility $\mathrm{Y}-\mathrm{Al}_{2} \mathrm{O}_{3} / \mathrm{SrTiO}_{3}$ heterostructure. EPL Europhys. Lett. 113, 47005 (2016).

47. Pippard, A. B. Magnetoresistance in metals. (Cambridge University Press, 1989).

48. Coey, J. M. D., Venkatesan, M. \& Stamenov, P. Surface magnetism of strontium titanate. J. Phys. Condens. Matter 28, 485001 (2016).

49. Chen, Y. Z. et al. A high-mobility two-dimensional electron gas at

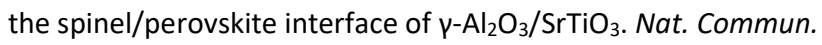
4, 1371 (2013).

50. Christensen, D. V. et al. Controlling the carrier density of $\mathrm{SrTiO}_{3}-$ based heterostructures with annealing. Adv. Electron. Mater. 1700026 (2017).
51. Schütz, P. et al. Microscopic origin of the mobility enhancement at a spinel/perovskite oxide heterointerface revealed by photoemission spectroscopy. Phys. Rev. B 96, 161409 (2017).

\section{Acknowledgements:}

We acknowledge the useful discussions with Raelph Claessen and Philipp Schütz from the University of Würzburg, Jeremy Levy from Pittsburgh University and $\mathrm{Yu}$ Zhang and Yulin Gan from the Technical University of Denmark. In addition, we thank Christian Bernhard and Meghdad Yazdi for insight in the temperature dependence of the carrier density extracted by infrared ellipsometry.

\section{Author Contributions:}

D.V.C., Y.F., N.P. and B.K. initiated this work. D.V.C. performed the transport measurements and prepared the GAO/STO samples. Y.X., Z.C., Y.H. and H.Y.H. prepared the LAO/STO samples. D.V.C., Y.F. and B.K. performed the scanning SQUID measurements. D.V.C., and Y.F. performed data analysis. D.V.C., Y.F., A.S., Y.Z.C., L.K., N.P. and B.K. interpreted the data. D.V.C. wrote the manuscript with Y.F. and input from all authors.

\section{Additional information:}

Supplementary information is available in the online version of the paper. Reprints and permissions information is available online at www.nature.com/reprints. Correspondence and requests for materials should be addressed to B. Kalisky (beena@biu.ac.il) and N. Pryds (nipr@dtu.dk), respectively.

\section{Competing Financial Interests:}

The authors declare no competing financial interests. 